\title{
Marsha Sharp: A Modern-day Pioneer
}

$\mathrm{T}$ he early advancement of the dietetic profession in Canada is often depicted through the careers of Violet Ryley and Kathleen Jeffs, 2 great women who exemplified the ideals of the profession by charting new directions. A reflection of the modern-day evolution of dietetics must now be portrayed through the influence Marsha Sharp has had on our profession.

Marsha's dietetics career began in 1977 in patient care and moved quickly to clinical management. Her profound appreciation for the role nutrition could make in people's lives was evident when she was chosen in the mid-1980s to lead the Canadian Dietetic Association (CDA) as Executive Director. Marsha's 33-year legacy in transforming CDA had begun. Marsha envisioned a Canada where dietitians had a national voice and influenced the shape of healthcare decisions. This vision led to the 1997 creation of Dietitians of Canada (DC) through a milestone merger of the national and provincial dietetic associations. As the CEO of the new organization, Marsha quickly became known for her dedication to advancing the profession and DC on local and international stages as well as for her systems thinking and innovative mind. Her commitment to results that mattered for the profession never wavered through a time of tremendous change in health service delivery, consumer interest in nutrition, technology, government lobbying, and the need for a networked organization.

Early in her career at DC, Marsha helped shape the landscape of practice-based dietetic research in Canada by championing the belief in its capacity to make dietetics stronger and more credible. Her vision led to the establishment of the Canadian Foundation for Dietetic Research in 1991. It remains today as the only research foundation dedicated to funding dietetic practice-based research.

Marsha was instrumental in fostering collaboration on the international stage as a founding member of the International Confederation of Dietetic Associations (ICDA). She served as Secretary since the inception in 2000 and now serves as Chair of the Board. Her vision for this organization was a collective voice for national dietetic associations where dietitians could practice free of borders. ICDA today consists of 40 national dietetic associations and 180,000 dietetic professionals. Andy Burman, CEO, British Dietetic Association, acclaims Marsha for "creating the environment that has seen the international community grow and collaborate in ways that other professions admire and envy."

Marsha is also celebrated globally for her drive to champion evidence-based standards and resources to support dietitians wherever they live and work. Her ability to recognize the potential synergy of seemingly unrelated ideas and to foster unique partnerships created the conditions that led to the online global nutrition knowledge base Practice-based Evidence in Nutrition $(\mathrm{PEN})$ in 2005. Today the PEN system is an unparalleled international resource with 6 global partners and tens of thousands of subscribers. Claire Hewat, CEO, Dietitians Association of Australia, attributes Marsha's mutual respect and collaboration to a beneficial relationship between the 2 associations in many areas, but none more important than the highly valued PEN collaboration.

Marsha's commitment to professional education led to the development of DC's distance learning platform at a time when such an approach was neither commonplace nor simple. She understood the need for all dietitians-wherever they were in the country-to have access to affordable, current, and evidence-based learning throughout their careers. Her deep conviction to quality dietetic education also supported the establishment of the Partnership for Dietetic Education and Practice in 2009, a collaborative interorganizational network dedicated to enabling excellence in dietetic education and practice.

Marsha was also an early adopter. Under her direction, DC was a front-runner in implementing a virtual workplace long before it became common business practice. She also recognized the power of embracing new technology, which led to the early creation of the DC website as a modern tool for reaching the Canadian public, and as a hub for the profession's voice and service to members. These insights were well ahead of the curve of other health professions at the time.

After 3 decades, Marsha officially retires from her DC role to spend more time with her husband Gord and to renew her many interests. Congratulations and thank you Marsha for all that you have done for our profession.

(Can J Diet Pract Res. 2017;78:217)

(DOI: $10.3148 /$ cjdpr-2017-032)

Published at dcjournal.ca on 16 November 2017

\section{Acknowledgements:}

Heather Wile, MSc, RD, FDC, was the narrator of this brief tribute to Marsha Sharp that represents reflections from many Canadian and international colleagues who worked closely with her on pivotal milestones. Repeatedly their reflections celebrated Marsha for her dedication to the profession, her thoughtful leadership, her collaborative approach and visionary mind, and her role in amplifying the visibility of dietitians around the world. 


\section{Marsha Sharp : une pionnière des temps modernes}

$\mathrm{L}$ es premiers avancements de la profession de diététiste au Canada sont souvent relatés en faisant référence aux carrières de $\mathrm{M}^{\text {mes }}$ Violet Ryley et Kathleen Jeffs, deux grandes femmes qui se sont illustrées en traçant de nouvelles voies pour la profession. Aujourd'hui, c'est en examinant l'influence qu'a eue Marsha Sharp sur notre profession que l'on peut procéder à une réflexion sur l'évolution actuelle de la diététique.

La carrière en diététique de Marsha a débuté en 1977 dans le domaine des soins aux patients. Peu de temps après, Marsha a toutefois axé sa carrière sur la gestion clinique. Au milieu des années 1980, lorsqu'elle a été choisie pour diriger l'Association canadienne des diététistes (ACD) en tant que directrice générale, son appréciation du rôle de la nutrition dans la vie des gens était manifeste. C'est donc à ce moment que débuta le legs qu'elle nous laisse aujourd'hui, à savoir de 33 années vouées à la transformation de l'ACD. Marsha avait une vision : un Canada où le discours des diététistes aurait une portée nationale et façonnerait les décisions en matière de soins de santé. Cette vision a mené à la création des Diététistes du Canada (DC) en 1997 grâce à une fusion historique des associations de diététistes nationale et provinciales. En tant que directrice générale de cette nouvelle organisation, Marsha s'est rapidement fait connaître pour son dévouement envers l'avancement de la profession et des DC, tant au niveau local qu'international, ainsi que pour sa pensée systémique et son esprit novateur. Au fil des années, son engagement envers des résultats significatifs pour la profession n'a jamais été ébranlé, malgré les grands changements survenus sur le plan de la prestation des services de santé, de l'intérêt des consommateurs pour la nutrition, des technologies, des efforts de lobbying auprès du gouvernement et de la nécessité d'établir une organisation en réseau.

Au début de sa carrière au sein des DC, Marsha a contribué à façonner le paysage canadien de la recherche en diététique fondée sur la pratique en défendant avec conviction la capacité de cette recherche à rendre la diététique plus forte et plus crédible. En 1991, ses efforts ont porté leurs fruits avec la création de la Fondation canadienne de la recherche en diététique, qui demeure aujourd'hui la seule fondation pour la recherche vouée exclusivement au financement de la recherche en diététique fondée sur la pratique.

En outre, Marsha a joué un rôle déterminant dans la promotion de la collaboration internationale à titre de membre fondatrice de l'International Confederation of Dietetic Associations (ICDA). Dès la création de cette association en 2000, elle y a occupé le rôle de secrétaire, puis est devenue présidente du conseil d'administration un rôle qu'elle occupe encore aujourd'hui. Elle envisageait cette organisation comme une voix collective pour les associations nationales de diététistes, un endroit où les diététistes pourraient pratiquer au-delà des frontières. L'ICDA regroupe aujourd'hui 40 associations nationales de diététistes et 180000 professionnels de la diététique. Andy Burman, directeur général de la British Dietetic Association, salue Marsha pour la «création de l'environnement qui a vu la communauté internationale croître et collaborer d'une manière qui suscite l'admiration et l'envie d'autres professions ».

Marsha est également reconnue mondialement pour sa détermination à promouvoir les normes et les ressources fondées sur des données probantes visant à appuyer les diététistes, partout où ils vivent et travaillent. Sa capacité à reconnaître la synergie potentielle entre des idées en apparence non liées et à favoriser des partenariats uniques a permis de réunir les conditions ayant mené en 2005 à la création de la base de connaissances internationale en ligne connue sous le nom de Pratique en nutrition reposant sur des données probantes (PEN). Aujourd'hui, le système PEN est une ressource internationale unique regroupant six partenaires mondiaux et des dizaines de milliers d'abonnés. Claire Hewat, directrice générale de la Dietitians Association of Australia, indique que c'est en raison de l'importance qu'accorde Marsha au respect mutuel et la collaboration que la relation est aussi bénéfique entre les deux associations, et ce, dans de nombreux domaines. Or, aucune collaboration n'a été aussi importante que celle entourant PEN.

L'engagement de Marsha envers la formation professionnelle a donné naissance à la plate-forme d'apprentissage à distance des DC à un moment où une telle approche n'était ni courante ni simple. Elle a compris qu'il fallait que tous les diététistes, peu importe où ils se trouvaient au pays, aient accès à un apprentissage abordable, actuel et fondé sur des données probantes tout au long de leur carrière. Par ailleurs, sa ferme conviction que la formation en diététique se doit d'être de la plus grande qualité a également soutenu l'établissement, en 2009, du Partenariat pour la formation et la pratique en nutrition, un réseau interorganisationnel formé dans le but de créer des occasions de collaboration et voué à l'excellence dans la formation et la pratique en nutrition.

Marsha est également une adopteuse précoce. Sous sa direction, les DC ont été avant-gardistes dans la mise en œuvre d'un milieu de travail virtuel, bien avant que cela ne devienne une pratique courante dans les entreprises. Elle a aussi su reconnaître la puissance des nouvelles technologies, ce qui a mené à la création précoce du site Web des DC à titre d'outil moderne pour atteindre le public canadien et de portail pour la voix de la profession et le service aux membres. Or, ces idées étaient bien en avance par rapport à celles d'autres professions de la santé.

Après trois décennies, Marsha quitte officiellement ses fonctions au sein des DC afin de passer plus de temps avec son mari, Gord, et de s'adonner à ses nombreuses passions. Félicitations, Marsha, et merci pour tout ce que vous avez fait pour notre profession.

(Rev can prat rech diétét. 2017; 78:218)

(DOI: $10.3148 /$ cjdpr-2017-032)

Publié au dcjournal.ca le 16 novembre 2017

\section{Remerciements}

Heather Wile, MSc, RD, FDC, était la narratrice de ce bref hommage à Marsha Sharp qui illustre les réflexions de nombreux collègues canadiens et internationaux ayant travaillé en étroite collaboration avec Marsha à la réalisation d'importants jalons. Leurs réflexions saluaient invariablement Marsha pour son dévouement envers la profession, son leadership avisé, son approche collaborative et son esprit visionnaire ainsi que pour son rôle dans l'amélioration de la visibilité des diététistes dans le monde entier. 